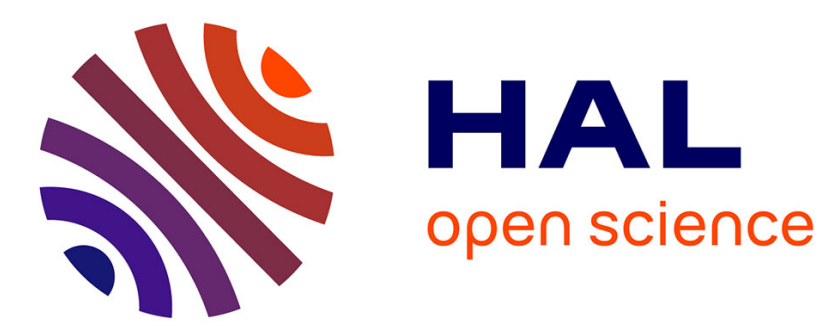

\title{
Théorie simplifiée des procédés de transfert inductif à haut rendement
}

\author{
C. Rioux
}

\section{To cite this version:}

C. Rioux. Théorie simplifiée des procédés de transfert inductif à haut rendement. Revue de Physique Appliquée, 1975, 10 (2), pp.75-79. 10.1051/rphysap:0197500100207500 . jpa-00243883

\section{HAL Id: jpa-00243883 https://hal.science/jpa-00243883}

Submitted on 1 Jan 1975

HAL is a multi-disciplinary open access archive for the deposit and dissemination of scientific research documents, whether they are published or not. The documents may come from teaching and research institutions in France or abroad, or from public or private research centers.
L'archive ouverte pluridisciplinaire HAL, est destinée au dépôt et à la diffusion de documents scientifiques de niveau recherche, publiés ou non, émanant des établissements d'enseignement et de recherche français ou étrangers, des laboratoires publics ou privés. 
Classification

Physics Abstracts

0.660

\title{
THÉORIE SIMPLIFIÉE DES PROCÉDÉS DE TRANSFERT INDUCTIF A HAUT RENDEMENT
}

\author{
C. RIOUX \\ Laboratoire de Physique des Plasmas (*) \\ Université de Paris-Sud, Centre d'Orsay, Bâtiment 214, 91405 Orsay, France
}

(Reçu le 26 septembre 1974)

\begin{abstract}
Résumé. - A l'aide d'un dispositif constitué uniquement par un système d'inductances et de conjoncteurs-disjoncteurs, il est possible de transférer avec un bon rendement l'énergie d'une inductance vers une charge quelconque. L'emploi marginal de condensateurs de faible capacité permet d'obtenir un rendement très élevé.
\end{abstract}

Abstract. - A system using only inductances and opening and closing switches has been developed to transfer energy from a storage inductance to any given load with a very high efficiency. Even higher efficiencies can be achieved if a number of small capacitors are used.

1. Introduction. - Une des tendances actuelles de l'électrotechnique impulsionnelle de grande puissance consiste à remplacer progressivement les lourdes et onéreuses batteries de condensateurs par des dispositifs beaucoup plus légers à base de stockage inductif $[1,2$, 3]. Cependant, des problèmes considérables restent à résoudre. Ils ont trait essentiellement au transfert de l'énergie stockée vers la charge d'utilisation. Ce transfert doit être rapide et s'effectuer avec un haut rendement, quelle que soit la charge. Si la rapidité du transfert peut être obtenue par l'utilisation de disjoncteurs spéciaux $[4,5]$, le problème de l'obtention de rendements élevés reste néanmoins entier.

Le but du présent article est d'examiner ce problème et de proposer une solution de portée générale. En associant un stockage inductif à un système convenable de commutateurs, il est en effet possible de créer une source d'énergie à rendement de transfert élevé dont l'emploi est particulièrement souple, même pour de hauts niveaux de puissance.

L'exposé présenté ici ne donne que les principes généraux de la conception de ces sources d'énergie. Les calculs détaillés sont rapportés par ailleurs $[6,7]$.

2. Position du problème. - Considérons un ensemble physique constitué par trois éléments : une inductance $\mathrm{L}$, un disjoncteur $\mathrm{A}$ et une charge $\mathrm{Ch}$ (voir Fig. 1). A l'instant initial, le disjoncteur A est fermé et aucun courant ne circule dans la charge. Si l'on ouvre le disjoncteur A, un courant égal circulera dans l'inductance $\mathrm{L}$ et la charge $\mathrm{Ch}$ et une fraction au moins de

(*) Laboratoire associé au C. N. R. S.

REVUE DE PHYSIQUe APPLIQUÉE. - T. 10, No 2, MARS 1975

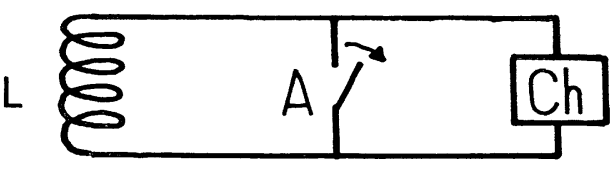

Fig. 1.

l'énergie stockée initialement dans L sera transférée vers Ch [4]. On montre aisément que le rendement du transfert d'énergie dépend de la nature de la charge. Les charges constituées par une résistance ou une inductance nulle au départ sont rares, tandis que les charges capacitives ne sont pas envisageables pour les questions de prix évoquées plus haut. Aussi, il reste à résoudre le problème consistant à obtenir un rendement de transfert élevé dans tous les autres cas, au besoin en intercalant entre $\mathrm{L}$ et $\mathrm{Ch}$ un dispositif $\mathrm{T}$ plus complexe que $\mathrm{A}$ (Fig. 2). Pour définir la structure à donner à $\mathrm{T}$, nous examinerons tout d'abord le cas où la charge est une inductance fixe ; nous étendrons ensuite l'étude au cas général.

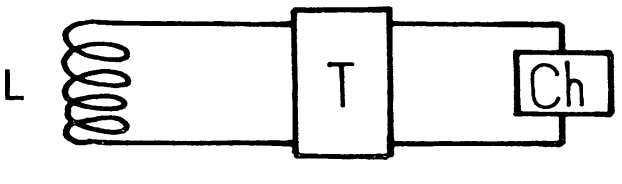

FIG. 2.

3. Transferts d'énergie simple entre deux inductances fixes. - Considérons le montage idéalisé de la figure 3, où l'inductance de stockage et la charge sont toutes 


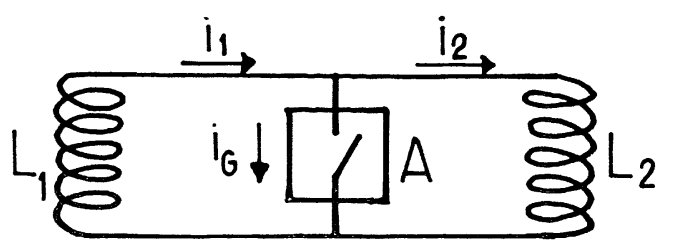

FIG. 3.

deux des inductances pures de valeurs respectives $L_{1}$ et $L_{2}$ et dont les résistances internes sont nulles.

Pendant le transfert d'énergie, les inductances $L_{1}$ et $L_{2}$ sont parcourues respectivement par les courants $i_{1}$ et $i_{2}$, tandis que l'interrupteur $\mathrm{A}$ est soumis à un courant $i_{\mathrm{G}}$ tel que $i_{\mathrm{G}}=i_{1}-i_{2}$. A l'instant initial, $i_{\mathrm{G}}=i_{1}$ tandis qu'en fin de transfert $i_{\mathrm{G}}=0$.

Au cours de l'évolution, on a :

$$
L_{1} \frac{\mathrm{d} i_{1}}{\mathrm{~d} t}=-L_{2} \frac{\mathrm{d} i_{2}}{\mathrm{~d} t} \quad \text { ou } \quad L_{1} i_{1}+L_{2} i_{2}=\mathrm{C}^{\mathrm{t}} .
$$

Pour préciser les aspects énergétiques du fonctionnement, appelons $W_{1}=\frac{1}{2} L_{1} i_{1}^{2}$ et $W_{2}=\frac{1}{2} L_{2} i_{2}^{2}$ les énergies stockées à chaque instant dans les inductances et $W_{\mathrm{T}}=W_{1}+W_{2}$ l'énergie totale. En introduisant le paramètre d'évolution $\theta$, on peut écrire :

$$
\left.\begin{array}{l}
i_{1}=\sqrt{\frac{2}{L_{1}}} \sqrt{W_{\mathrm{T}}} \cos \theta \\
i_{2}=\sqrt{\frac{2}{L_{2}}} \sqrt{W_{\mathrm{T}}} \sin \theta
\end{array}\right\} .
$$

A l'aide des variables $\sqrt{W_{1}}$ et $\sqrt{W_{2}}$, on peut représenter simplement l'état instantané du système et son évolution (voir Fig. 4).

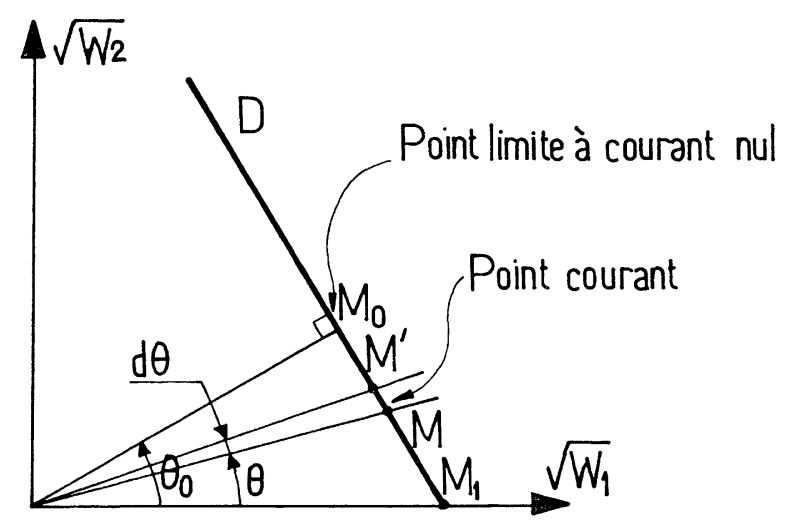

FIG. 4. - Diagramme représentatif du transfert de l'énergie entre deux inductances.

D'après (1), le lieu du point courant $M$ est une droite $\mathrm{D}$ dont la normale est définie par sa longueur $O M_{0}$ et par sa position $\theta_{0}$ telle que :

$$
\operatorname{tg} \theta_{0}=\sqrt{\frac{L_{2}}{L_{1}}}
$$

L'évolution complète du système de la figure 3 est alors représentable par le segment $M_{1} M_{0}$ de la figure 4. (La différence des deux courants s'annule progressivement lorsque $M$ tend vers $M_{0}$.) Le rendement global du transfert vaut :

$$
\eta=\frac{\left(W_{2}\right)_{\text {final }}}{\left(W_{1}\right)_{\text {initial }}}=\frac{1}{4} \sin ^{2} 2 \theta_{0} .
$$

L'on voit d'après (4) que $\eta$ ne peut excéder ainsi $25 \%$, lequel rendement est obtenu pour $L_{1}=L_{2}$ (c'est-à-dire pour $\theta_{0}=\pi / 4$ ).

Afin de préciser les conditions de transfert de l'énergie, explicitons le rendement $\eta_{\theta}$ d'un transfert élémentaire entre deux points $M$ et $M^{\prime}$ voisins définis par les angles $\theta$ et $\theta+\mathrm{d} \theta$. On obtient :

$$
\eta_{\theta}=-\frac{\mathrm{d} W_{2}}{\mathrm{~d} W_{1}}=\frac{\operatorname{tg} \theta}{\operatorname{tg} \theta_{0}} .
$$

Par suite, ces transferts élémentaires d'énergie sont d'autant meilleurs que le point courant $M$ est plus proche de la position finale $M_{0}$ où $i_{1}=i_{2}$. Cette constatation permet d'entrevoir les conditions d'un transfert inductif global à haut rendement $:$ il faut que le point $M$ représentatif de chaque transfert élémentaire soit proche du pied $M_{0}$ de la perpendiculaire menée de $\mathrm{O}$ sur D. Or, cette condition n'est réalisable que si l'on peut obtenir à chaque instant du transfert une position de $M_{0}$ proche de $M$, ce qui est impossible dans le dispositif de la figure 3 .

Cette impossibilité est due à la fixité de la droite $\mathrm{D}$, dont la pente ne dépend que du rapport constant $L_{2} / L_{1}$; pour obtenir un bon rendement dans toutes les phases du transfert, il faudrait faire varier progressivement le rapport $L_{2} / L_{1}$, c'est-à-dire au moins une des inductances.

4. Transferts d'énergie entre inductances variables. A partir des considérations précédentes, on peut imaginer un dispositif plus perfectionné, constitué d'une inductance variable $L_{2}$ associée à une inductance fixe $L_{1}$ comme représenté sur la figure 5 .

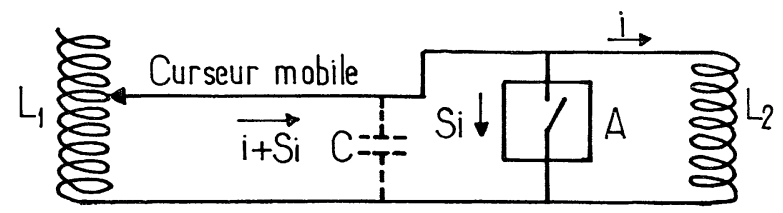

FIG. 5. - Dispositif simple de transfert à haut rendement avec interrupteur et curseur.

Le fonctionnement s'effectue à l'aide d'une suite de séquences où l'on ne transfère à chaque fois qu'une faible énergie dans les conditions d'un bon rendement. Chaque séquence s'effectue en trois temps (voir Fig. 6). Dans le premier, A est initialement fermé, les inductances $L_{1}$ et $L_{2}$ sont constantes et les courants qui circulent en leur intérieur diffèrent de la valeur petite 


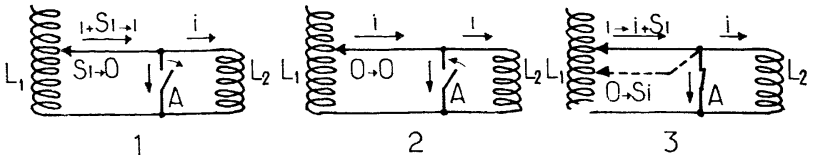

FIG. 6. - Décomposition d'une séquence élémentaire de transfert.

$\delta_{\mathrm{i}} ;$ l'ouverture progressive de $\mathrm{A}$ annule $\delta_{\mathrm{i}}$. Dans un deuxième temps, on ferme $\mathrm{A}$. Dans le troisième, $\mathrm{A}$ reste fermé et l'on fait varier l'inductance $L_{1}$ sans modifier son énergie : il en résulte à nouveau un déséquilibre $\delta_{\mathbf{i}}$ des courants circulant dans les deux inductances, ce qui rétablit les conditions initiales du premier temps. Cette dernière opération qui peut paraître difficile s'effectue facilement si toutes les spires constituant l'inductance $L_{1}$ sont entre elles en mutuelle totale.

Le transfert complet par séquences successives est représentable sur la figure 7 . Chaque séquence à induc-

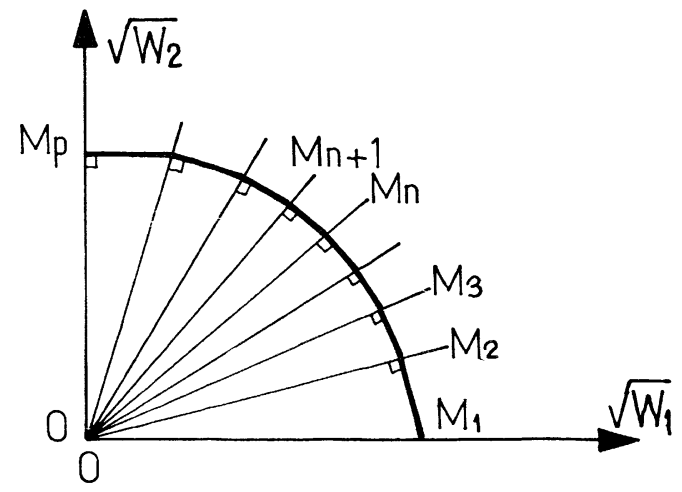

Fig. 7. - Diagramme représentatif d'un transfert complet à séquences multiples avec disjoncteur.

tance constante est représentée par un petit segment de droite $\overrightarrow{M_{n} M_{n+1}}$ perpendiculaire à $\overrightarrow{O M_{n+1}}$. L'examen direct de la figure montre que si le nombre de séquences croît, la suite des points $M_{1}, M_{2}, \ldots, M_{p}$ se rapproche d'un cercle. Alors $\left|\overrightarrow{O M_{p}}\right|=\left|\overrightarrow{O M_{1}}\right|$ : le rendement global tend vers 1 .

Si l'on effectue une suite de $p$ séquences, on montre aisément que le rendement maximum est obtenu lorsque les points $M_{n}$ sont équidistants. Il vaut :

$$
\eta=\left(\cos \frac{\pi}{2 p}\right)^{2 p}
$$

(Un transfert simple correspond au cas $p=2$.) La figure 8 qui représente la fonction $\eta=\eta(p)$ montre toutefois qu'un excellent rendement exige un nombre de séquences assez élevé, ce qui complique le dispositif ; nous reviendrons plus loin sur cette difficulté.

Finalement, nous sommes parvenu à effectuer un transfert à haut rendement, mais au prix d'une inductance variable. Remarquons tout d'abord que si le dispositif à curseur est matérialisé par un système d'inter-

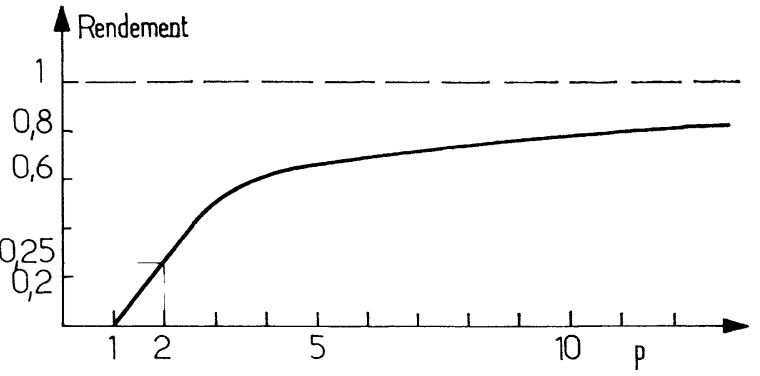

FIG. 8. - Rendement d'un transfert en fonction du nombre de séquences.

rupteurs, A peut être supprimé. On aboutit au montage de la figure 9.

D'autre part, si l'on désire absolument que les inductances $L_{1}$ et $L_{2}$ restent constantes, on peut obtenir

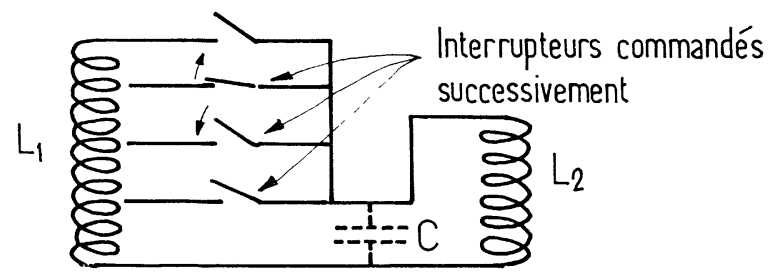

Fig. 9. - Dispositif de transfert à curseur constituéd'un ensemble de conjoncteurs-disjoncteurs.

encore un transfert à haut rendement. Il suffit de placer entre $L_{1}$ et $L_{2}$ un dispositif complexe $\mathrm{T}$ comportant deux bobines à inductance variable en mutuelles totales entre elles. $\mathrm{T}$ est alors un transformateur à rapport de transformation variable (voir Fig. 10).

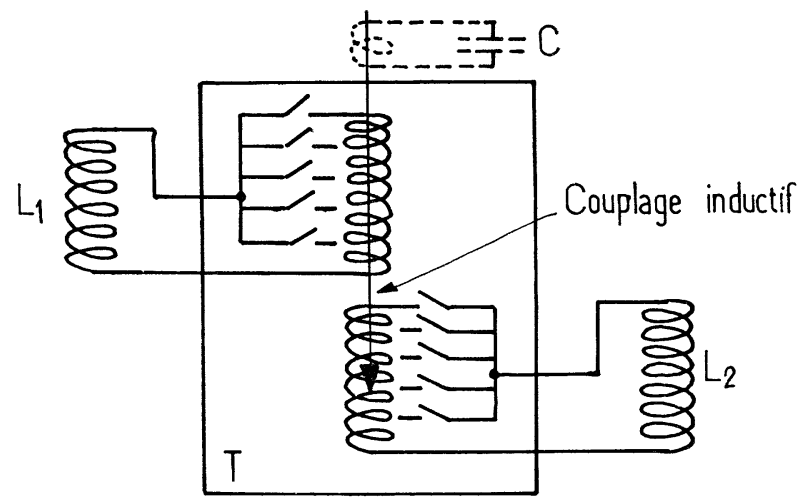

FIG. 10. - Dispositif de transfert entre deux inductances quelconques fixes.

5. Transferts d'énergie non dissipatifs. - Dans le dispositif de la figure 10 , la solution au problème du transfert inductif a été obtenue par une suite de séquences dissipatrices au niveau des interrupteurs successifs. Le rendement global ne devient excellent 
que pour $p$ grand (Fig. 8). On peut alors se poser la question suivante : n'existe-t-il pas des dispositifs non dissipatifs qui permettent d'obtenir directement un transfert avec un rendement égal à 1 ?

Certes, une solution bien connue au niveau d'un transfert simple consiste à remplacer $\mathrm{A}$ par un condensateur C (voir Fig. 11). A l'instant initial, le condensa-

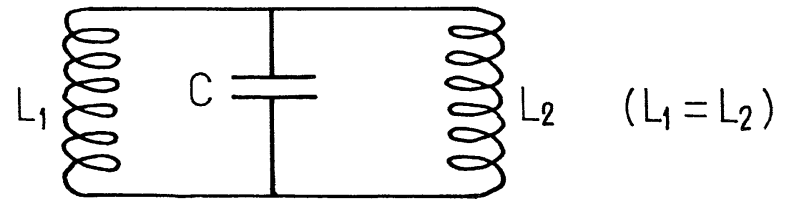

FIG. 11. - Dispositif de transfert à condensateurs.

teur n'est pas chargé et aucun courant ne circule dans $L_{2}$. Au cours du fonctionnement, on assiste à un mouvement pendulaire de l'énergie entre les deux inductances, le condensateur stockant lui-même de l'énergie transitoirement au cours de chaque transfert. Dans ce cas, la représentation de l'évolution dans un diagramme en $\left(\sqrt{W_{1}}, \sqrt{W_{2}}\right)$ montre que le point $\mathrm{M}$ figurant l'état du système parcourt indéfiniment de manière périodique le segment de droite $M_{1} M_{2}$ ayant $M_{0}$ pour centre (voir Fig. 12). Il est à remarquer que si $L_{1} \neq L_{2}$,

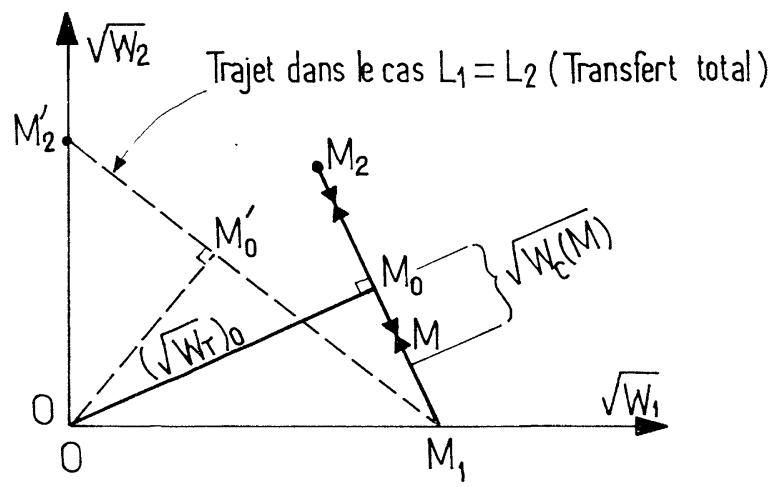

FIG. 12. - Diagramme représentatif d'un transfert simple réversible avec condensateurs.

le transfert de l'énergie entre les deux inductances n'est jamais total. L'énergie maximale stockée transitoirement dans le condensateur est représentée par $\left(\overline{M_{0} M_{1}}\right)^{2}$.

Cette énergie électrostatique est trop élevée pour qu'une telle solution soit envisagée en électrotechnique impulsionnelle. Cependant, on peut s'en inspirer pour perfectionner les transferts dissipatifs à séquences multiples que nous avons déjà étudiés. Pour cela, il suffit d'associer aux montages des figures 5, 9 ou 10 un condensateur $\mathrm{C}$ de faible capacité ( $\mathrm{C}$ est représenté en pointillé sur ces figures).

Pour expliquer le fonctionnement avec condensateur, nous allons considérer le montage particulier de la figure 5 en remplaçant le disjoncteur A par le condensateur $\mathrm{C}$.

Au début de la séquence (voir Fig. 13), le courant

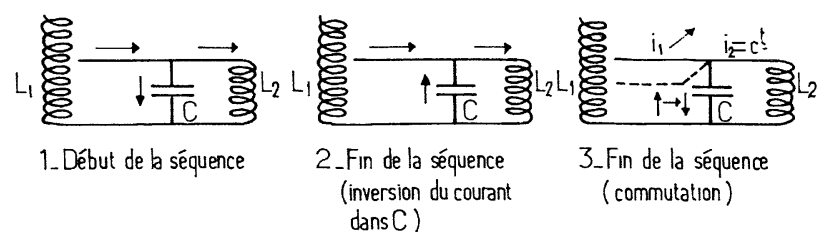

FIG. 13. - Détail de la séquence élémentaire d'un transfert réversible avec condensateur.

dans $L_{1}$ est supérieur au courant dans $L_{2}$ et le condensateur C est déchargé ; le point $M$ représentant l'état du système est en $M_{1}$ (voir Fig. 14). Au cours de l'évolution qui suit, le condensateur se charge jusqu'à une valeur maximale atteinte lorsque $M$ est en $M_{0}$, puis se décharge à nouveau. Par analogie avec le cas rapporté

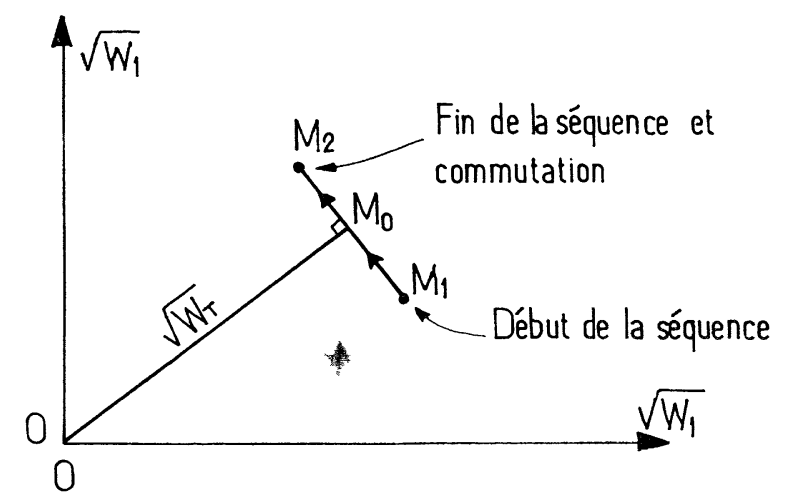

Fig. 14. - Diagramme représentatif d'un transfert élémentaire avec condensateur.

sur la figure 12 , le point $M$ franchit la limite $M_{0}$; il atteint le point $M_{2}$ symétrique de $M_{1}$ lorsque la charge est nulle à nouveau. A ce dernier instant, de l'énergie a été transférée de $L_{1}$ à $L_{2}$ avec un rendement égal à 1 , mais le courant circulant dans $L_{1}$ est devenu inférieur au courant dans $L_{2}$. Pour rétablir un état semblable à l'état initial $\left(i_{1}>i_{2}\right)$, on déplace le curseur d'un plot. Le transfert complet de l'énergie s'effectue alors par une suite de séquences régulières représentées sur la figure 15 .

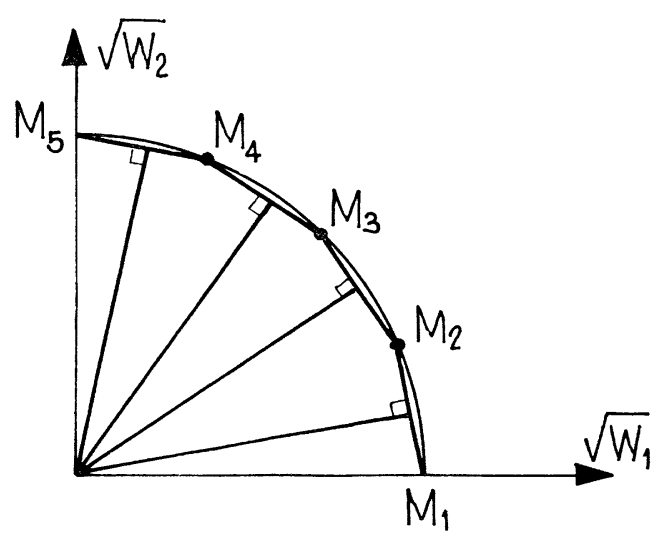

FIG. 15. - Diagramme représentatif d'un transfert complet réversible à séquences multiples (avec condensateur). 
Si $p$ est le nombre de ces séquences, on montre que l'énergie maximale dans le condensateur vaut :

$$
W_{\mathrm{c}}=W_{0} \sin ^{2} \frac{\pi}{4 p} .
$$

En examinant la figure 8, on voit que pour $p$ de l'ordre de 10, l'utilisation d'un condensateur dont l'énergie maximale est inférieure à $1 \%$ de l'énergie transférée, fait passer le rendement de transfert de 0,75 à 1 . L'emploi de condensateurs en quantité modérée permet donc d'améliorer sensiblement les performances des transferts inductifs. Notons aussi que la réduction conjointe de l'énergie à dissiper dans les disjoncteurs favorise beaucoup leur fonctionnement.

Si l'on désire là encore transférer l'énergie directement d'une inductance fixe à une autre inductance fixe, on peut placer entre $L_{1}$ et $L_{2}$ un dispositif transformateur à rapport variable $\mathrm{T}$ analogue à celui de la figure 10. Le condensateur sera couplé inductivement au transformateur comme indiqué sur cette figure.

6. Transferts sur une charge quelconque. - Après avoir étudié les transferts entre deux inductances, on peut se proposer d'extrapoler l'étude à une charge quelconque. Remarquons tout d'abord que l'utilisation rationnelle d'une source d'énergie nécessite une bonne adaptation des impédances du générateur et de la charge. Pour une source classique tels que des condensateurs, la forme de la rafale d'énergie qui est déterminée par l'impédance de la charge se confond très rarement avec une bonne utilisation de l'énergie. Au contraire, pour une source du type inductance-transformateur variable, il est toujours possible de choisir le débit idéal de l'énergie par une loi temporelle convenable des variations du nombre de spires.

Plus précisément, appelons $n=n(t)$ le rapport de transformations du transformateur $\mathrm{T}$ de la figure 10 , $P=P(t)$ la puissance fournie à chaque instant à la charge et $i=i(t)$ le courant correspondant. On montre [6] que si $P(t)$ et $i(t)$ sont deux fonctions données a priori, il existe toujours une loi de variation $n=n(t)$ qui permet de les obtenir conjointement tout en conservant un rendement élevé :

$$
n(t)=\frac{1}{\sqrt{2}} \frac{1}{\sqrt{\log i(t)-\int_{0}^{t} \frac{P(t)}{L_{1} i^{2}(t)} \cdot \mathrm{d} t}} .
$$

Par suite, les ensembles inductance-transformateur variable constituent des sources d'énergie particulièrement souples qui sont adaptables de manière parfaite à toutes les charges; cette souplesse est supérieure à celle de toutes les sources habituelles.

7. Applications. - Nos études ont surtout été effectuées dans le cadre de l'électrotechnique impulsionnelle. En employant des contacteurs rapides à faible inductance et des disjoncteurs à feuilles explosées, chaque séquence de commutation peut être effectuée en quelques microsecondes $[8,9,10]$. Il en résulte que les propriétés que nous venons de décrire peuvent être obtenues dès que les temps caractéristiques du fonctionnement sont supérieurs à quelques dizaines de microsecondes.

Toujours dans le cadre de l'électrotechnique impulsionnelle, le problème de la récupération avec rendement de l'énergie stockée dans une inductance est alors résolu et les applications correspondantes peuvent être importantes :

- Alimentations pulsées à récupération d'énergie de grandes bobines pour accélérateurs de particules et pour machines à plasma à confinement magnétique (Tokamak, $\theta$-pinch droits ou toniques, etc.).

- Création de champs pulsés intenses dans de grands volumes durant quelques millisecondes.

- Alimentations modulées de lasers de puissance ou de souffleries à rafales.

- Formage magnétique de pièces de très grandes dimensions.

8. Conclusion. - L'étude que nous venons d'effectuer montre que l'association d'une inductance fixe et d'un dispositif transformateur de rapport variable résout le problème de la récupération de l'énergie stockée dans une inductance. Les sources d'énergie ainsi constituées sont particulièrement souples d'emploi ; elles sont adaptables à une gamme très large de charges d'utilisation et libèrent leur énergie avec un rendement proche de l'unité.

Utilisées en électrotechnique impulsionnelle, elles sont $a$ priori susceptibles de fournir des rafales d'énergie élevées (par exemple de l'ordre du MJ ou beaucoup plus) dont la forme peut être fixée dès que le temps de l'impulsion dépasse une centaine de microsecondes.

Une étude plus détaillée tenant compte des résistances et des inductances de fuite des divers circuits utilisés a été menée par ailleurs [11]; elle confirme les conclusions de notre étude générale simplifiée.

\section{Bibliographie}

[1] Hassel, W. F., ARS Space Power Systems Conf., Santa Monica, Calif. sept. 25-28 (1962).

[2] Gaignebet, E., Stockage d'énergie pour alimenter une soufflerie à arc, ONERA no $1 / 1428$ PN (1968).

[3] Shearer, J. W. et al., J. Appl. Phys. 39 (1968) 2102.

[4] Rioux, C., Rioux-Damidau, F., Revue Phys. Appl. 9 (1974) 539.

[5] Dimarco, J. N., Burkhardt, L. C., J. Appl. Phys. 41 (1973) 3894.
[6] Rioux, C., Rapport Interne, avril 1971.

[7] Rioux, C., Rapport Interne, septembre 1971.

[8] Antoni, B., Revue Phys. Appl. 9 (1974) 331.

[9] Bleys, C. A., Lebely, D., Rioux-Damidau, F., Rev. Sci. Instrum., à paraître.

[10] Henins, I., Marshall, J., Rev. Sci. Instrum. 39 (1968) 1481.

[11] Legentil, M., Rapport Interne LF 18 (1973). 